\title{
RECENT TRENDS IN THE FIELD OF JORDAN-BANACH ALGEBRAS
}

\author{
BERNARD AUPETIT \\ Département de mathématiques et de statistique, Université Laval \\ Québec, Canada, G1K $7 P_{4}$
}

1. Introduction. A Jordan algebra is a non-associative algebra in which the product satisfies the two conditions $x y=y x$ and $(x y) x^{2}=x\left(y x^{2}\right)$, for all $x, y$ in the algebra. Such algebras were introduced, in the period 1932-1934, by P. Jordan, J. von Neumann and E. Wigner in order to improve the quantum mechanics formalism (see [24]). In the subsequent years they were studied by A. A. Albert $[1,2]$ who gave a complete classification of finite-dimensional Jordan algebras. Since that time Jordan algebras have been intensively investigated by algebraists and the bibliography on this subject is very huge (see for instance $[22$, $23,34])$.

Given an associative algebra $A$ we can build a Jordan algebra, denoted by $A^{+}$, defining the Jordan product $x \cdot y=\frac{1}{2}(x y+y x)$. Any Jordan subalgebra of $A^{+}$is consequently a Jordan algebra. Such algebras are called special algebras.

Another interesting example is built in the following way. Let $V$ be a linear vector space and let $\varphi$ be a symmetric bilinear form on $V$. We define a product on $J=\mathbb{C} \times V$ by

$$
(\alpha, x) \cdot(\beta, y)=(\alpha \beta+\varphi(x, y), \beta x+\alpha y) .
$$

Then $J$ is a Jordan algebra for this product and every element $u=(\alpha, x)$ is algebraic of degree two because it satisfies the equation $u^{2}-2 \alpha u-\varphi(x, x)=0$. This algebra is in fact a special algebra (see [34], Exercise 1, p. 57). Unfortunately, there are exceptional algebras, that is to say, non-special algebras and this is what mainly makes the difficulty of the theory (see for instance Albert's theorem on $H\left(C_{3}\right)$ in [34], pp. 55-57).

1991 Mathematics Subject Classification: 17C65, 46H70, 46K70, 46L70.

The paper is in final form and no version of it will be published elsewhere. 
On a Jordan algebra $A$ we define the important quadratic operator $U_{a}$ defined by $U_{a}(x)=2 a(a x)-a^{2} x$. It has the following property:

$$
U_{x} U_{y} U_{x}=U_{U_{x}(y)}, \quad \text { for } x, y \in A .
$$

N. Jacobson introduced the notion of invertibility in Jordan algebras, which of course generalizes the standard notion of invertibility in associative algebras. Given $x$ in $A$ we say that $x$ is invertible if there exists $y$ in $A$ such that $x y=1$ and $x^{2} y=x$. This element $y$ is unique and is denoted by $x^{-1}$. This notion is intimately related with the quadratic operator.

THEOREM 1.1. If $x \in A$ then $x$ is invertible if and only if $U_{x}$ is invertible in $L(A)$, the algebra of linear operators on $A$, in which case $U_{x^{-1}}=\left(U_{x}\right)^{-1}$. If $x, y \in A$, then they are both invertible if and only if $U_{x}(y)$ is invertible in $A$. In particular, $x$ is invertible if and only if $x^{n}$ is invertible for every integer $n \geq 1$.

This theorem implies that the set of invertible elements in $A$ is invariant under taking powers but unfortunately, it is not stable for the product.

2. Analytic properties of the spectrum. A Jordan-Banach algebra A is a Jordan algebra with a complete norm satisfying $\|x y\| \leq\|x\|\|y\|$, for $x, y$ in $A$. Adjoining an identity to $A$ if necessary, we may suppose without loss of generality that $A$ has an identity, denoted by 1 , and that $\|1\|=1$. Because we shall be concerned mainly with analytical tools we shall suppose throughout that $A$ is a complex algebra with identity. Very often the study of real Jordan-Banach algebras can be reduced to the study of complex ones using complexification and the identity principle for analytic functions, but in some cases this study may be very tricky.

As seen before, $x$ is invertible if and only if $U_{x}$ is invertible and $U_{x^{-1}}=\left(U_{x}\right)^{-1}$. This implies the following.

THEOREM 2.1. The set $U$ of invertible elements of $A$ is open and is invariant under the differentiable homeomorphism $x \rightarrow x^{-1}$.

For $x$ in $A$ we can define the spectrum of $x$, denoted by $\operatorname{Sp} x$, to be the set of $\lambda \in \mathbb{C}$ such that $\lambda 1-x$ is not invertible in $A$. The spectral radius $r(x)$ is by definition the maximum of $|\lambda|$ for all $\lambda$ in the spectrum of $x$. As for Banach algebras, it is possible to obtain the following.

THEOREM 2.2. Let $x$ be an element of a Jordan-Banach algebra. Then

(i) $\operatorname{Sp} x$ is compact and non-empty,

(ii) $\lambda \rightarrow(\lambda 1-x)^{-1}$ is analytic on the complement of $\operatorname{Sp} x$,

(iii) $r(x)=\lim _{n \rightarrow \infty}\left\|x^{n}\right\|^{1 / n}$.

The standard holomorphic functional calculus in Banach algebras extends to Jordan-Banach algebras. This comes from the fact that the closed subalgebra generated by 1 and $x$ is associative, and consequently is a Banach algebra. 
Theorem 2.3 (Holomorphic Functional Calculus). Let A be a Jordan-Banach algebra, $x$ in $A$ and $U$ a neighbourhood of the spectrum of $x$. If $h$ is holomorphic on $U$ then we can define

$$
h(x)=\frac{1}{2 \pi i} \int_{\Gamma} h(\lambda)(\lambda 1-x)^{-1} d \lambda
$$

where $\Gamma$ is a positively oriented curve included in $U$ and surrounding $\operatorname{Sp} x$. Then we have the following properties:

(i) $h(x)$ is independent of the choice of $\Gamma$ lying in $U$ and surrounding $\operatorname{Sp} x$,

(ii) $\varphi: h \rightarrow h(x)$ is an algebraic morphism from $H(U)$ into the smallest closed strongly associative subalgebra containing 1 and $x$; moreover, if $h(\lambda)=1$ then $\varphi(h)=1$ and if $h(\lambda)=\lambda$ then $\varphi(h)=x$

(iii) $\varphi$ is continuous on $H(U)$ for the uniform convergence on every compact subset of $U$,

(iv) $\operatorname{Sp} h(x)=h(\operatorname{Sp} x)$.

For a detailed exposition of this theorem see [26]. Until the beginning of the 1980 s, the previous three theorems were the only analytic spectral tools known in the theory of Jordan-Banach algebras.

The next result is the fundamental tool which gives us the opportunity to use systematically the deep theory of analytic multifunctions (for more details on analytic multifunctions see Chapter VII of [6]).

Theorem 2.4 (B. Aupetit and A. Zraïbi [12]). Let $\lambda \rightarrow f(\lambda)$ be an analytic function defined on an open subset $D$ of the complex plane with values in a Jordan-Banach algebra. Then the multifunction $\lambda \rightarrow \operatorname{Sp} f(\lambda)$ is analytic.

This result implies in particular that the functions $\lambda \rightarrow \log r(\lambda), \lambda \rightarrow$ $\log \delta_{n}(f(\lambda))$ and $\lambda \rightarrow \log c(f(\lambda))$ are subharmonic, where $\delta_{n}$ and $c$ denote respectively the $n$th diameter and the capacity of the spectrum of $f(\lambda)$. This theorem has a huge number of consequences, some of them elementary, like the analogues of Newburgh's theorems on spectral continuity or like Corollaries 2.5 and 3.1 below, and some of them very deep.

Corollary 2.5 ([12]). Let A be a Jordan-Banach algebra having a subadditive (or uniformly continuous) spectral radius $r$. Then for every $x$ in $A$ and every bounded derivation $D$ of $A$ we have $r(D x)=0$.

The proof uses subharmonicity and the fact that $e^{\lambda D}$ is an automorphism of $A$. It can be used with $D=R_{a} R_{b}-R_{b} R_{a}$ where $R_{a}(x)=a x$.

Other consequences are the following two results.

Theorem 2.6 (B. Aupetit and M. A. Youngson [11]). Let A be a JordanBanach algebra with involution $*$. The following properties are equivalent:

(i) if $h=h^{*}$ then $\operatorname{Sp} h \subset \mathbb{R}$, 
(ii) if $h=h^{*}$ and $k=k^{*}$ have positive spectra then so has $h+k$,

(iii) $x^{*} x$ has positive spectrum for every $x$ in $A$.

We recall that $A$ is a $J B^{*}$-algebra if for every $x$ in $A$ we have $\left\|U_{x}\left(x^{*}\right)\right\|=\|x\|^{3}$. It is easy to check that if $A$ is a $J B^{*}$-algebra and $h=h^{*}$ then $\|\exp (i h)\|=1$ and the converse is true.

Corollary 2.7 ([11]). A is a $\mathrm{JB}^{*}$-algebra for an equivalent norm if and only if there exists a constant $C \geq 1$ such that $\|\exp (i h)\| \leq C$ for all $h$ satisfying $h=h^{*}$.

$J B^{*}$-algebras are an important tool which has been systematically studied (see [21] for more details).

Theorem 2.4 is also important for philosophical reasons. It says that for any analytic function $f$ with values in a Jordan-Banach algebra the multifunction $\lambda \rightarrow \operatorname{Sp} f(\lambda)$ is analytic. But, by a famous representation theorem due to Z. Słodkowski, every analytic multifunction can be locally represented as $\operatorname{Sp} T(\lambda)$ where $T$ is an analytic function with values in the algebra of bounded operators on the Hilbert space $\ell^{2}$ (see [6], p. 167); so, in some sense, Theorem 2.4 reduces a non-associative problem to an associative one. It is the reason for the following creed which has never been contradicted yet: any Banach algebra result which is proved by a purely spectral argument must be true and proved in a similar way, modulo some technical modifications, in the situation of Jordan-Banach algebras.

The first revolutionary application will be given in $\S 3$. We proved it in 1982 in order to extend B. Johnson's theorem on equivalence of complete norms.

3. The radical. The notion of Jacobson radical for associative algebras has been generalized by K. McCrimmon to Jordan algebras (see [27, 28]). In a Jordan algebra we say that an ideal $I$ is quasi-invertible if for every $x \in I$ we have $1-x$ invertible. McCrimmon proved that in any Jordan algebra there exists a unique maximal quasi-invertible ideal. By definition this ideal is the Jacobson radical of $A$ and is denoted by $\operatorname{Rad} A$. McCrimmon proved that if $A$ is a special Jordan algebra then the radical coincides with the associative Jacobson radical. From this definition it is easy to see that the radical of $A / \operatorname{Rad} A$ is zero, in other words, this quotient algebra is semisimple (some authors say "semiprimitive").

Using Corollary 2.5 we can easily get the following:

Corollary 3.1 ([12]). Let $A$ be a Jordan-Banach algebra. Then $A / \operatorname{Rad} A$ is associative (consequently, a commutative Banach algebra) if and only if $r$ is subadditive and submultiplicative on $A$.

By Theorem 3.5 below we shall see that the same result is true if $r$ is only supposed to be subadditive (for the associative situation see Theorem 5.2.2 of [6]). For a semisimple Banach algebra having a derivation $D$ which satisfies $r(D x)=0$ for all $x$ in $A$ it is known that $D=0$. Is the same true for Jordan-Banach algebras if $D$ is supposed to be at least bounded? 
In 1967, using representation theory, B. E. Johnson proved that for a semisimple Banach algebra all complete algebra norms are equivalent. This result implies in particular that any involution on such an algebra is continuous. These results have very important consequences in the associative theory. Johnson's argument depends heavily on representation theory and on the fact that for Banach algebras the Jacobson radical is the intersection of the kernels of irreducible representations, that is, primitive ideals. Unfortunately, in the case of Jordan-Banach algebras the similar result is not true (as noticed by J. M. Osborn [29]) so Johnson's argument cannot be adapted (except in some rather easy situations like $H^{*}$-algebras).

At first sight the extension of Johnson's result to Jordan-Banach algebras seemed to be impossible to get at the end of the 1970s as mentioned by J. Martínez Moreno in the introduction of his thesis [26].

Finally, in [4] we proved a beautiful extension of Johnson's result for Banach algebras (Theorem 5.5.1 of [6]), and in the case of Jordan-Banach algebras the following.

THEOREM 3.2. A semisimple Jordan-Banach algebra has a unique complete norm topology and every Jordan epimorphism from a Jordan-Banach algebra onto a semisimple Jordan-Banach algebra is continuous.

COROLlaRY 3.3. Every involution on a semisimple Jordan-Banach algebra is continuous.

These two results simplify considerably the results and proofs of [31]. The argument of the proof is very simple, but based on subharmonicity of $\log r(f(\lambda))$ which derives from Theorem 2.4 and Liouville's theorem for subharmonic functions.

At that time we were not able to give Johnson's extension in full generality for non-associative algebras, because we had no equivalent of Zemánek's characterization of the radical (Theorem 5.3.1 in [6]) which was, until 1992, based on representation theory. But now there is a spectral characterization of the radical for Jordan-Banach algebras (see Theorem 3.5 below). Consequently, we have

TheOrem 3.4. Let $A$ and $B$ be two Jordan-Banach algebras. Suppose that $T$ is a linear mapping from $A$ into $B$ which is spectrally contractive, that is, $r(T x) \leq r(x)$ for every $x$ in $A$. Denoting by $\mathcal{S}(T)$ the separating space of $T$ (that is, the set of $y$ in $B$ for which there exists $x_{n}$ in $A$ converging to 0 such that $T x_{n}$ converges to $\left.y\right)$ then $y \in \mathcal{S}(T)$ implies $r(T x) \leq r(y+x)$, for all $x$ in $A$. In particular, if $T$ is onto we have $\mathcal{S}(T) \subset \operatorname{Rad} B$, so $T$ is continuous if $B$ is semisimple.

In [7] we gave a purely spectral proof of Zemánek's characterization of the radical in the case of Banach algebras. Using Theorem 2.4 and sophisticated arguments on analytic multifunctions we succeeded in proving the following. 
THEOREM 3.5 (Spectral Characterization of the Radical). Let a be an element of a Jordan-Banach algebra $A$. Then $a$ is in the radical of $A$ if and only if $\sup \{r(x+t a): t \in \mathbb{C}\}$ is finite for every $x$ in $A$.

Corollary 3.6. Let a be an element of a Jordan-Banach algebra A. Then a is in the radical of $A$ if and only if $r\left(U_{x}(a)\right)=0$ for every $x$ in $A$.

Corollary 3.7. Let a be an element of a Jordan-Banach algebra A. Then a is in the radical of $A$ if and only if there exists $C \geq 0$ such that $r(x) \leq C\|x-a\|$ on a neighbourhood of $a$.

If $A$ is a semisimple Jordan algebra N. Jacobson has proved that $U_{a}=0$ for some $a \in A$ implies $a=0$. In the case of semisimple Jordan-Banach algebras this result comes immediately from the previous corollary because $\left(U_{x}(a)\right)^{2}=$ $U_{U_{x}(a)}(1)=U_{x} U_{a} U_{x}(1)=U_{x} U_{a}\left(x^{2}\right)=0$, so $r\left(U_{x}(a)\right)=0$ for every $x$ in $A$; consequently, $a$ is in the radical of $A$, hence it is zero.

For a semisimple Banach algebra it is known that every derivation is bounded. This beautiful result due to B. E. Johnson and A. Sinclair is proved using representation theory. It would be very interesting to give a purely spectral proof of that result, probably using analytic arguments as we did in the proof of Theorem 3.4 , because it would give some hope to solve the same problem for semisimple Jordan-Banach algebras.

4. Spectrally finite Jordan-Banach algebras. In 1954, I. Kaplansky proved the following result: if $\phi$ is a ring morphism from a semisimple Banach algebra $A$ onto a Banach algebra $B$, then $A$ admits a decomposition $A=I_{1} \oplus I_{2} \oplus I_{3}$ where $I_{1}, I_{2}, I_{3}$ are closed two-sided ideals of $A$ such that $I_{1}$ is finite-dimensional, $\phi$ is linear on $I_{2}$ and $\phi$ is antilinear on $I_{3}$. The proof is based on the result that a semisimple Banach algebra for which every element has finite spectrum is finite-dimensional. This result has been generalized in many directions (see [3], Chapter 3, for applications and historical comments, and also [6], Chapter V, $\S 4$, for a more modern treatment).

This result suggested to us the study of the same problem in the situation of Jordan-Banach algebras. The same result cannot be true in general because if we take an infinite-dimensional Banach space $V$ with Jordan product defined on $\mathbb{C} \times V$ using a symmetric bilinear form, as explained in the introduction, then $A=\mathbb{C} \times V$ is a Jordan-Banach algebra for which every element has a spectrum with at most two points. The first attempt in this direction is the following result.

THEOREM $4.1([12])$. Let $U$ be a non-empty open subset of a Jordan-Banach algebra $A$. Suppose that for every $x$ in $U$ the spectrum of $x$ has one point. Then $A / \operatorname{Rad} A=\mathbb{C}$.

We suggested to A. Kaïdi the study of this question and it was solved by his student M. Benslimane. The final result is the following. 
Theorem 4.2 (M. Benslimane and A. Kaïdi $[15,13])$. Let $U$ be a non-empty open subset of a semisimple Jordan-Banach algebra A. Suppose that for every $x$ in $U$ the spectrum of $x$ is finite. Then we have a finite decomposition $A=$ $I_{1} \oplus \ldots \oplus I_{n}$ where the $I_{k}$ are simple closed ideals of $A$ having one of the following two properties:

(i) $I_{k}$ is finite-dimensional,

(ii) $I_{k}$ is infinite-dimensional and the Jordan product on $\mathbb{C} 1 \oplus I_{k}$ is defined by a symmetric bilinear form.

With this last result is it possible to extend Kaplansky's theorem to ring morphisms of Jordan-Banach algebras?

5. The socle. If $A$ is a complex Banach algebra the socle, denoted by $\operatorname{Soc} A$, is by definition the sum of all minimal left (resp. right) ideals of $A$. Equivalently, the socle is the sum of all left ideals (resp. right ideals) of the form $A p$ (resp. $p A$ ) where $p$ is a minimal idempotent, that is, $p^{2}=p$ and $p A p=\mathbb{C} p$. If $A$ is finite-dimensional then $A$ coincides with its socle. If $A=B(X)$, the algebra of bounded operators on a Banach space $X$, the socle of $A$ consists of finite-rank operators. For more details about socle see [3], pp. 78-87 and [6], pp. 110-113.

For Jordan-Banach algebras the definition of the socle is slightly more difficult. To simplify we shall suppose that the Jordan-Banach algebra $A$ is semisimple.

We say that an idempotent $p$ is minimal if $U_{p}(A)=\mathbb{C} p$, in which case $U_{p}(A)$ is a minimal quadratic ideal. But there is another type of minimal quadratic ideals $Q$, those which have the properties $Q=U_{q}(A)$ for every $q \in Q$ and $Q^{2}=0$ (this derives from a more general result of N. Jacobson showing that in any Jordan algebra there are only three types of minimal quadratic ideals).

By definition the socle of $A$, denoted by $\operatorname{Soc} A$, is the sum of all minimal quadratic ideals of $A$.

Theorem 5.1 (J. M. Osborn and M. L. Racine [30]). The socle of $A$ is an ideal and it is the sum of all ideals generated by minimal projections.

It is not clear at all for which algebras the socle is non-zero. In the case of Banach algebras, B. A. Barnes proved that the socle is non-zero if every element of the algebra has a finite or countable spectrum. His argument is very technical, in particular it uses Shilov's idempotent theorem, a rather difficult theorem using several complex variables. In [6], Theorem 5.7.8, we gave a purely subharmonic proof which can be adapted to the situation of Jordan-Banach algebras.

Theorem 5.2 (B. Aupetit and L. Baribeau [9]). Let $A$ be a semisimple Jordan-Banach algebra. Suppose that every element of A has a finite or countable spectrum. Then A has non-zero socle.

It is even enough to suppose that the spectrum is finite or countable on an absorbing subset of $A$. 
Extending the corresponding associative concept which was mainly studied by B. A. Barnes we can define a modular annihilator Jordan-Banach algebra $A$ as a semisimple Jordan-Banach algebra such that $A / \operatorname{Soc} A$ is radical. M. Benslimane and A. Rodríguez Palacios, using the ideas contained in [5] or in [6], Chapter V, $\S 7$, extended former results of B. A. Barnes. A. Fernández López obtained similar results but his arguments are very technical.

Theorem 5.3 (M. Benslimane and A. Rodríguez Palacios [16], A. Fernández López [17]). Let $A$ be a semisimple Jordan-Banach algebra such that the spectrum of every element has at most zero as a limit point. Then $A$ is modular annihilator.

From Theorems 4.2 and 5.3 we get the next structure theorem.

TheOrem 5.4 (B. Aupetit and L. Baribeau [9]). Let A be a separable JordanBanach algebra. Suppose that every element of $A$ has a finite or countable spectrum. Then there exists an ordinal number $\alpha_{0}$ of the first or second class and a composition sequence $\left(I_{\alpha}\right)_{\alpha \leq \alpha_{0}}$ of closed ideals such that $I_{0}=\operatorname{Rad} A, I_{\alpha_{0}+1}=A$ and $I_{\alpha+1} / I_{\alpha}$ is modular annihilator for $\alpha \leq \alpha_{0}$.

For the definition of first and second class ordinal numbers, see the book of W. Sierpiński [33]. The $I_{\alpha}$ are in fact the transfinite hypersocles obtained from the successive $\alpha$-Calkin algebras.

A. Fernández López and A. Rodríguez Palacios [19] proved that for a semisimple Jordan-Banach algebra $A$ the socle coincides with the largest von Neumann regular ideal. Given a semisimple Jordan-Banach algebra A. Fernández López [18] proved that its socle is an algebraic ideal and conversely if $I$ is any algebraic ideal then every element of $I$ can be written as the sum of an element of the socle and a nilpotent element. This result was improved by M. Benslimane, O. Jaa and A. Kaïdi [14] who proved that every element of a spectrally finite ideal can be written as the sum of an element of the socle and an element whose square is zero. Using recent results of O. Loos [25] it is even possible to prove much more.

Theorem 5.5 (M. Benslimane, O. Jaa, A. Kaïdi and O. Loos). Let $A$ be a semisimple Jordan-Banach algebra and let I be a spectrum finite ideal of $A$. Then $I$ is included in the socle of $A$.

For more comments on this question see [32], p. 40 and pp. 100-101, and [25].

In [10], B. Aupetit and H. du T. Mouton gave a purely spectral characterization of the socle in the case of Banach algebras which has very interesting consequences. Using sophisticated results on analytic multifunctions it is possible to prove the following four results.

Theorem 5.6. Let $A$ be a semisimple Jordan-Banach algebra, let $a \in A$ and let $n$ be a non-negative integer. The following conditions are equivalent:

(i) $\operatorname{Sp}\left(U_{x}(a) \backslash\{0\}\right)$ has at most $n$ points for every $x$ in $A$,

(ii) $\bigcup_{t \in F} \operatorname{Sp}(y+t a) \subset \mathrm{Sp} y$ for every $y$ in $A$ and $F$ a finite subset of $\mathbb{C}$ having at most $n+1$ non-zero points. 
We denote by $\mathcal{F}_{n}$ the set of $a \in A$ having the above two properties. By Corollary 3.6, $\mathcal{F}_{0}$ is the radical of $A$, hence $\{0\}$. We shall say that $\mathcal{F}_{n}$ is the set of elements of $A$ with rank less than or equal to $n$. The main result (Theorem 5.10) says that $\bigcup_{n \geq 0} \mathcal{F}_{n}$ coincides with the socle of $A$.

Theorem 5.7. If $a \in \mathcal{F}_{n}$ then $U_{x}(a) \in \mathcal{F}_{n}$ for every $x \in A$.

The proof of Theorem 5.9 needs a beautiful geometrical characterization of algebraic varieties of $\mathbb{C}^{2}$ we have recently proved and which was apparently unknown before. The symbol \# denotes cardinality.

Lemma 5.8. Let $V \neq \mathbb{C}^{2}$ be a closed subset of $\mathbb{C}^{2}$ above $\mathbb{C}$ which has the following properties:

(a) $\mathbb{C}^{2} \backslash V$ is a pseudoconvex open set,

(b) for every $\lambda \in \mathbb{C}$ we have either $\#((\{\lambda\} \times \mathbb{C}) \cap V) \leq m$ or $\{\lambda\} \times \mathbb{C} \subset V$,

(c) for every $\mu \in \mathbb{C}$ we have either $\#((\mathbb{C} \times\{\mu\}) \cap V) \leq n$ or $\mathbb{C} \times\{\mu\} \subset V$.

Then $V$ is a complex algebraic subvariety of $\mathbb{C}^{2}$ of degree at most $m+n$.

From that we get the following

TheOREM 5.9. If $a \in \mathcal{F}_{m}$ and $b \in \mathcal{F}_{n}$ then $a+b \in \mathcal{F}_{m+n}$.

Using Theorems 5.5, 5.7 and 5.9 we immediately get the next result.

TheOREM 5.10. $\bigcup_{n \geq 0} \mathcal{F}_{n}$ coincides with the socle of $A$.

All these theorems, which will appear in [8], imply the existence of an additive trace on the socle whose restriction to $\mathcal{F}_{n}$ is continuous. They also imply that every element of the socle of $A$ is a finite sum of elements of $\mathcal{F}_{1}$. Another consequence is that a linear mapping from a semisimple Jordan-Banach algebra onto itself which preverves the spectrum leaves the $\mathcal{F}_{n}$ invariant and consequently the socle.

To finish this paper we would say that there remains the fascinating task to try to understand more clearly the intimate correlation of Jordan-Banach algebras, homogeneous symmetric spaces and analytic multifunctions.

The reader intending to know more about the recent developments of the algebraic theory of Jordan algebras has to read the recent big survey of A. Rodríguez Palacios [32].

\section{References}

[1] A. A. Albert, On Jordan algebras of linear transformations, Trans. Amer. Math. Soc. 59 (1946), 524-555.

[2] —, A structure theory for Jordan algebras, Ann. of Math. 48 (1947), 446-467.

[3] B. Aupetit, Propriétés spectrales des algèbres de Banach, Lecture Notes in Math. 735 Springer, Heidelberg, 1979.

[4] - The uniqueness of the complete norm topology in Banach algebras and Banach-Jordan algebras, J. Funct. Anal. 47 (1982), 1-6. 
[5] B. Aupetit, Inessential elements in Banach algebras, Bull. London Math. Soc. 18 (1986), 493-497.

[6] -, A Primer on Spectral Theory, Universitext, Springer, New York, 1991.

[7] - Spectral characterization of the radical in Banach and Jordan-Banach algebras, Math. Proc. Cambridge Philos. Soc. 114 (1993), 31-35.

[8] - Spectral characterization of the socle in Jordan-Banach algebras, to appear.

[9] B. Aupetit et L. Baribeau, Sur le socle dans les algèbres de Jordan-Banach, Canad. J. Math. 41 (1989), 1090-1100.

[10] B. Aupetit and H. du T. Mouton, Spectrum-preserving linear mappings in Banach algebras, Studia Math. 109 (1994), 91-100.

[11] B. Aupetit and M. A. Youngson, On symmetry of Banach-Jordan algebras, Proc. Amer. Math. Soc. 91 (1984), 364-366.

[12] B. Aupetit et A. Zraïbi, Propriétés analytiques du spectre dans les algèbres de JordanBanach, Manuscripta Math. 38 (1982), 381-386.

[13] M. Benslimane, A. Fernández López et A. Kaïdi, Caractérisation des algèbres de Jordan-Banach de capacité finie, Bull. Sci. Math. (2) 112 (1988), 473-480.

[14] M. Benslimane, O. J a and A. Kaïdi, The socle and the largest spectrum finite ideal, Quart. J. Math. Oxford (2) 42 (1991), 1-7.

[15] M. Benslimane et A. Kaïdi, Structure des algèbres de Jordan-Banach non commutatives complexes régulières ou semi-simples à spectre fini, J. Algebra 113 (1988), 201-206

[16] M. Benslimane et A. Rodríguez Palacios, Caractérisation spectrale des algèbres de Jordan-Banach non commutatives complexes modulaires annihilatrices, J. Algebra 140 (1991), 344-354.

[17] A. Fernández López, Modular annihilator Jordan algebras, Comm. Algebra 13 (1985), $2597-2613$.

[18] - Noncommutative Jordan Riesz algebras, Quart. J. Math. Oxford (2) 39 (1988), 67-80.

[19] A. Fernández López and A. Rodríguez Palacios, On the socle of a noncommutative Jordan algebra, Manuscripta Math. 56 (1986), 269-278.

[20] - , - Primitive noncommutative Jordan algebras with nonzero socle, Proc. Amer. Math. Soc. 96 (1986), 199-206

[21] H. Hanche-Olsen and E. Størmer, Jordan Operator Algebras, Pitman, New York, 1984.

[22] N. Jacobson, Structure and Representations of Jordan Algebras, Amer. Math. Soc. Colloq. Publ. 39, Amer. Math. Soc., Providence, R.I., 1968.

[23] —, Lectures on Quadratic Jordan Algebras, Tata Institute of Fundamental Research, Bombay, 1969 .

[24] P. Jordan, J. von Neumann and E. Wigner, On an algebraic generalization of the quantum mechanical formalism, Ann. of Math. 35 (1934), 29-64.

[25] O. Loos, Properly algebraic and spectrum-finite ideals in Jordan systems, Math. Proc. Cambridge Philos. Soc. 114 (1993), 149-161.

[26] J. Martínez Moreno, Sobre álgebras de Jordan normadas completas, Tesis doctoral, Universidad de Granada, 1977.

[27] K. McCrimmon, The radical of a Jordan algebra, Proc. Nat. Acad. Sci. U.S.A. 62 (1969), 671-678.

[28] -, A characterization of the radical of a Jordan algebra, J. Algebra 18 (1971), 103-111.

[29] J. M. Osborn, Representations and radicals of Jordan algebras, Scripta Math. 29 (1973), 297-329.

[30] J. M. Osborn and M. L. Racine, Jordan rings with nonzero socle, Trans. Amer. Math. Soc. 251 (1979), 375-387.

[31] P. S. Putter and B. Yood, Banach-Jordan *-algebras, Proc. London Math. Soc. (3) 41 (1980), 21-44. 
[32] A. Rodríguez Palacios, Jordan structures in analysis, preprint.

[33] W. Sierpiński, Cardinal and Ordinal Numbers, PWN, Warszawa, 1958.

[34] K. A. Zhevlakov, M. Slin'ko, J. P. Shestakov and A. I. Shirshov, Rings That Are Nearly Associative, Academic Press, New York, 1982.

Editorial note: See also the paper of A. R. Sourour in this volume. 